CORRECTION

https://doi.org/10.1038/s41586-019-0950-8

\title{
Author Correction: Inference of ecological and social drivers of human brain-size evolution
}

Mauricio González-Forero \& Andy Gardner

Correction to: Nature https://doi.org/10.1038/s41586-018-0127-x, published online 23 May 2018; corrected online 28 June 2018.

In the Acknowledgements section of this Letter, the words "M.G.-F. was funded by a Marie Skłodowska-Curie Individual Fellowship (No 701464)" should read "This project has received funding from the European Union's Horizon 2020 research and innovation programme under the Marie Skłodowska-Curie grant agreement No 701464". 